\title{
Caracterización Tipológica del Sector Hotelero de la Provincia de Los Ríos, Ecuador
}

\section{Typological Characterization of Los Ríos Province, Ecuador, Hotel Industry}

\author{
${ }^{*}$ Guillermo Angamarca Izquierdo ${ }^{1}$, Edwin Cruz Rosero ${ }^{1}$, Javier Bucheli Espinoza ${ }^{1}$, Carlos \\ Martínez Medina ${ }^{1}$ \\ ${ }^{1}$ Universidad Técnica Estatal de Quevedo. Facultad de Ciencias Empresariales. Carrera de \\ Marketing. Campus "Ingeniero Manuel Haz Álvarez"Av. Quito, Km 1.5 vía a Santo Domingo de \\ Los Tsáchilas \\ *gangamarca@uteq.edu.ec
}

Fecha de recepción: 17/01/2018

Fecha de aceptación: 28/03/2018

Publicado: $29 / 06 / 2018$

\section{Resumen}

El objetivo general de este trabajo consiste en caracterizar tipológicamente el sector hotelero de la provincia de Los Ríos, Ecuador. Específicamente, se enfoca en las características tipológicas de ubicación, tamaño y categoría de los hoteles de Los Ríos. Se parte de la siguiente problematización: ¿Cuáles son las características de los hoteles de Los Ríos, en función de su tipología: Ubicación, tamaño y categoría? Para la recolección de la información relativa a las características tipológicas de los hoteles se emplea un censo, dirigido a los gerentes de los 41 establecimientos hoteleros de Los Ríos, registrados en el MINTUR, Dirección provincial de Los Ríos: Quevedo (24), Babahoyo (7), Montalvo (2), Vinces (3), Baba (1), Valencia (2), Mocache (1) y Pueblo Viejo (1). Los principales resultados de la investigación indican que el $81 \%$ de los hoteles de la provincia de los Ríos registrados en el MINTUR son de ubicación de ciudad, el 7 \% de naturaleza, el $12 \%$ son hoteles balnearios. No se registraron hoteles de aeropuerto, de playa, aparta-hoteles, albergues turísticos o moteles, en el sentido técnico de su clasificación. Por otro lado, El $100 \%$ de los hoteles de la provincia de los Ríos, registrados en el Ministerio de Turismo (MINTUR), pertenece a la clase de tamaño pequeño. El mayor grupo de los hoteles (41\%) de la 
provincia de los Ríos tiene la categorización de tres estrellas. Estos hoteles son muy utilizados por personas en turismo de negocios.

Palabras clave: Marketing de Servicios, Marketing Turístico, Hotelería.

\begin{abstract}
The general objective of this work is to typologically characterize Los Ríos province, Ecuador, hotel industry. Specifically, it focuses on the characteristics of location, size and category of hotels in Los Ríos. It starts on the following problem: What are the characteristics of the hotels in Los Ríos, depending on their type: Location, size and category? For collection of information regarding to the typological characteristics of the hotels, a census is used, directed to the managers of the 39 hotel establishments of Los Ríos, registered in Tourism Ministry (MINTUR), Provincial Direction of Los Ríos: Quevedo (24), Babahoyo (7), Montalvo (2), Vinces (3), Baba (1), Valencia (2), Mocache (1) and Pueblo Viejo (1). The main research results indicate that $81 \%$ of the hotels in the province of Los Ríos registered in the MINTUR are of city location, $7 \%$ of nature, $12 \%$ are spa hotels. There were no airport hotels, beach hotels, apart hotels, tourist hostels or motels, in the technical sense of their classification. On the other hand, $100 \%$ of the hotels in the province of Los Rios province registered in the MINTUR belong to the small size class. The largest group of hotels (41\%) of the province of Los Ríos province has the categorization of three stars. These hotels are widely used by business travelers.
\end{abstract}

Keywords: Services Marketing, Tourism Marketing, Hotel Industry.

\title{
Introducción
}

El turismo es una de las industrias de mayor demanda mundial. Según la Organización Mundial del Turismo (OMT, 2017), en el año 2016 esta demanda fue de 1 billón 220.000 
millones de dólares, que representa un 4,5\% más que en el 2015. En Ecuador, la demanda turística, en el año 2015, registró ingresos aproximados por 986 millones de dólares, 13\% más que en el 2014 (Proecuador, 2016). En este mismo año, en la provincia de Los Ríos, el turismo generó ingresos por 84796000 dólares, lo que constituye el $0.86 \%$ del total nacional.

Esta importante oferta turística se satisface con el apoyo directo de uno de sus componentes básicos: El sector hotelero. No obstante, este sector no se ha desarrollado enteramente. Su ciclo de vida aún está en la etapa de introducción; es incipiente. Particularmente, adolece de una caracterización técnica de los hoteles, en función de su tipología: Ubicación, tamaño y categoría, que posibilite análisis más profundos de su accionar. En este contexto, esta investigación se planteó los siguientes objetivos específicos: 1) Determinar la ubicación de los hoteles de la provincia de los Ríos; 2) establecer el tamaño de los hoteles de la provincia de los Ríos; y 3) categorizar los hoteles de la provincia de los Ríos, registrados en el MINTUR. Se propone como hipótesis específicas: 1. La ubicación o localización de los hoteles depende de la ciudad en la que se encuentra; 2. la categoría de los hoteles de los Ríos está condicionada por la ciudad en la que laboran.

Es indudable que para una correcta caracterización de los hoteles hay que considerar su tipología en función de su ubicación, su tamaño y su categoría (OMT, 2008). El sector hotelero comprende todos aquellos establecimientos que facilitan alojamiento con o sin servicios complementarios. Las variables empleadas para clasificar a los establecimientos hoteleros por distintos autores son más o menos similares. Estas variables hacen referencia principalmente a la localización, las instalaciones y servicios, tamaño, mercado objetivo, tipo de propiedad y categoría del hotel.” (Cortina, F., Martínez, A. y Várela, B., 2002). 
Bajo una perspectiva general, y en función del lugar en el que se encuentre ubicado el hotel, estos pueden ser urbanos o de ciudad, hoteles de aeropuerto, hoteles de playa, hoteles de naturaleza, hoteles de apartamento o aparta- hotel, albergues turísticos, hoteles balnearios y moteles, (si tienen recepción y botones pasa a denominarse motor hotel, generalmente con más de 50 habitaciones). La clasificación de los hoteles, en cuanto a su ubicación o localización tipológica, puede resultar compleja, ya que determinar si un hotel es de ciudad, de playa o de montaña, en ocasiones puede responder a diferentes criterios (Mellinas, J., Martínez, S. M., Bernal, J. J, 2014).

En función de su dimensión o tamaño los hoteles pueden ser: Pequeños (Menos de 100 habitaciones), medianos (de 100 a 250 habitaciones) y grandes (más de 250 habitaciones) (Espino - Rodríguez, T., y Gil- Padilla, A. 2014).

En cuanto a su categoría, básicamente, los hoteles se clasifican en hoteles de lujo, hoteles de nivel medio, hoteles que ofrecen servicios limitados y hoteles económicos. Los criterios de clasificación en todos los países están basados en la estructura del hotel, las instalaciones y servicios ofrecidos, aunque algunos países como Francia incluyen métodos más subjetivos como la calidad, el personal o facilidades a los clientes. En España la categoría de los hoteles está representada por estrellas. (Arcarons, R., Goitia, V., González, N., 2012). El lenguaje técnico turístico ha simplificado las diversas clasificaciones agrupando los hoteles en: Categoría lujo, categoría semilujo, categoría $1^{\mathrm{a}}$ superior, categoría $1^{\mathrm{a}}$, categoría turista o estándar (Esteban, A. y Reinares, 2008).

No obstante lo anotado, no existe un sistema homogéneo para todos los países. (González, M., 2010). En palabras de Vallen y Vallen (2002), “existen diferencias entre los hoteles europeos y los americanos". Los hoteles europeos se clasifican, según Lattin (2007), en "hoteles de lujo (cuatro y cinco estrellas), hoteles con categoría intermedia (tres y dos estrellas) y hoteles 
económicos (una estrella); mientras que en los hoteles americanos se distinguen en función de la localización, el precio, el nivel de servicio y los complementos ofrecidos en la habitación.

"Los hoteles de dos estrellas son establecimientos que tienen piezas con un baño y ducha. Son muy utilizados por personas en viajes de negocios. Los de tres estrellas, disponen de habitaciones más grandes con baño, ducha, artículos de baños y tocador; televisor, cable pagado y teléfono. El servicio es manejado por personas calificadas. Entregan alimentación completa y diversas bebidas y tragos. Los hoteles de cuatro estrellas ofrecen habitaciones más grandes, con mejor decoración y más equipados. El servicio es más profesional, con servicios a la habitación las 24 horas. Cuentan con lavandería y limpiezas en seco y con productos más refinados y elaborados." Los de cinco estrellas "cuentan con espacios amplios y lujosos, dirigidos a un estrato social alto. El inmueble y el mobiliario reflejan elegancia y sofisticación. El servicio es de la máxima calidad. El o los restaurantes tendrán calidad internacional. Las habitaciones contarán con todas las comodidades y tecnología posibles: equipos de música, jacuzzis, tv-cable, etc. También ofrecerán gimnasios y peluquería. Su ubicación es de las mejores, en todas las ciudades que se encuentran” (Lattin, 2007).

En resumen, Mestres (1999) considera que, “de modo general, pese a que resulta difícil hablar de una única clasificación de los establecimientos hoteleros, estos se caracterizan de acuerdo con su tipología: ubicación, tamaño y categoría." Esta es la caracterización que se emplea en esta investigación.

\section{Materiales y métodos}


Para la recolección de la información relativa a la caracterización de los hoteles de la provincia de Los Ríos, en función de su ubicación, tamaño y categorización, se empleó un censo, dirigido a los gerentes de los hoteles bajo estudio.

La investigación propuesta se llevó a cabo en la zona urbana de la provincia de Los Ríos, Ecuador, durante los meses de mayo y junio del 2018. Los Ríos están localizada en la región costa, en el centro del país. Tiene una superficie de 7.150,90 km2. Su población total es de 650.178 habitantes (335.279 hombres y 314.899 mujeres). Tiene la siguiente división políticoadministrativa (cantones): Baba, Babahoyo, Buena Fe, Mocache, Montalvo, Palenque, Puebloviejo, Quevedo, Quinsaloma, Urdaneta, Valencia, Ventanas y Vinces. Su Población Económicamente Activa (PEA) es de 224.451 (184.125 hombres y 40.326 mujeres). Índice de Desarrollo Humano (IDH): \#22 (último) en el Índice ODM-1 y el \#12 en el Índice ODM-2 (67,7\% pobreza por ingresos). Extrema pobreza por Necesidades Básicas Insatisfechas (NBI): $42,24 \%$.

La investigación se apoyó en la utilización de un conjunto de métodos empíricos y teóricos. En lo relativo a los métodos empíricos, se emplearon los históricos, a través de los que se hizo una recopilación de las fuentes secundarias/documentales sobre las investigaciones relacionadas con la tipología del sector hotelero, con el propósito de obtener la sistematización de los aspectos conceptuales y metodológicos acerca de la ubicación, el tamaño y la categoría de los hoteles. Se enfatizaron aspectos teóricos tales como: La caracterización tipológica de los hoteles.

También se emplearon los métodos genético (para investigar el origen de las variables que rigen el comportamiento relacionado del fenómeno), hipotético-deductivo (para encontrar la correlación científica entre las variables independientes del problema bajo investigación y sus variables dependientes, de modelación (representación artificial de una caracterización de los hoteles de manera que se brinde un resultado científico tipológica que permita corregir aquellos 
elementos que puedan ser susceptibles de cambio), descriptivo (para recolectar la información relativa a la caracterización tipológica de los hoteles (oferta), el mismo que representó una interacción directa con los directivos (oferta) de los hoteles seleccionados de modo transversal, y explicativo (procuró la identificación y análisis de las variables dependientes de la investigación, aplicadas a la investigación de campo, y sus resultados, expresados técnicamente en cuadros, gráficos y cifras estadísticas, relativas a la caracterización tipológica de los hoteles de la provincia de Los Ríos.

En lo que se refiere a los métodos teóricos, se empleó la revisión documental, que permitió documentar un marco teórico acerca de la caracterización tipológica de los hoteles. De este modo se revisaron, consultaron, analizaron y discutieron libros, artículos científicos y documentos técnicos -impresos o electrónicos- relacionadas con el estado del arte de la tipología del sector hotelero, con el propósito de obtener la sistematización de los aspectos conceptuales y metodológicos relacionados. También se revisaron los métodos estadísticos de comprobación y análisis más viables y acertados para el tratamiento de la información a obtener: Varianza y test de Chi Cuadrado. Además, se aplicó un método de observación, a través de una guía de observación previamente elaborada, acorde con los preceptos de la investigación científica, in situ, y de modo práctico y preliminar, para determinar cuál era el ambiente, la situación y las condiciones tangibles e intangibles de los hoteles bajo estudio, previo su caracterización tipológica, en virtud de visitas realizadas a ellos. Estas fueron realizadas previo la investigación de campo, mediante entrevistas informales y exploratorias.

Se emplearon también entrevistas formales (a los gerentes o administradores de los 41 hoteles bajo estudio) y un censo de los hoteles de la provincia de Los Ríos, a fin de caracterizarlos 
tipológicamente, para cuyo efecto se empleó un cuestionario pre-elaborado. Las variables bajo estudio que se consideraron para la caracterización de los hoteles de la provincia de Los Ríos fueron: "ubicación", "dimensión o tamaño" y "categoría" de los hoteles. Las respectivas variables y sus atributos se presentan en la Tabla 1.

Tabla 1.

Variables empleadas para la caracterización de los hoteles

\begin{tabular}{lllllllll}
\hline Variables & & & \multicolumn{5}{c}{ Atributos } \\
\hline Ubicación & Ciudad & Aeropuerto & De playa & $\begin{array}{l}\text { De } \\
\text { naturaleza }\end{array}$ & $\begin{array}{l}\text { Aparta- } \\
\text { hotel }\end{array}$ & $\begin{array}{l}\text { Albergue } \\
\text { turístico }\end{array}$ & $\begin{array}{l}\text { Hotel } \\
\text { balneario }\end{array}$ & Motel \\
Tamaño & Pequeño & Mediano & Grande & & & & \\
Categoría & Una & Dos & Tres & Cuatro & Cinco & & \\
& estrella & estrellas & estrellas & estrellas & estrellas & & \\
\hline
\end{tabular}

Elaboración: Los autores

\section{Diseño de la Investigación}

La recolección de la información necesaria para la evaluación de la investigación empezó con los estudios preliminares y exploratorios, los que dieron paso a la construcción del instrumento para el censo de los hoteles, en el que se empleó sendos cuestionarios. Fue necesario la realización del censo, dado el reducido número de hoteles bajo estudio (41), lo que no ameritaba una muestra.

A través de la información proporcionada por el censo, dirigido a los gerentes o administradores de los hoteles que componen el sector hotelero de la provincia de Los Ríos, se pudo realizar la caracterización de los hoteles, en función de su tipología, tamaño y categoría.

Los instrumentos que empleó la investigación fueron los cuestionarios pre-elaborados para el censo (ver Anexo 1). La recolección de los datos se la realizó sin influir el entorno propio (elementos tangibles, capacidad de respuesta, seguridad y empatía) de cada uno de los hoteles 
bajo estudio y sin manipular la información que se encontró en la investigación de campo (censo dirigido). Se consideró como población al conjunto de hoteles bajo observación, representado por los gerentes o administradores de los 41 hoteles registrados en el Ministerio de Turismo (MINTUR). Se los clasificó por cantones. En la Tabla 2 se presenta un condensado de esa información.

Tabla 2.

Identificación de hoteles por cantones de Los Ríos, 2017

\section{Cantones Nombre de los Hoteles}

Quevedo Almaquint, Aroma de cacao, Barros, Bravo, Casa Blanca, Central, Continental, Costa del sol, Ejecutivo, Europa, Flor de Líbano, Guayaquil, Hotel del Río, Imperial, La Española, Olímpico, Palmar del Sol, Presidente, Rancho San Vinicio, Riverside, San Andrés, Sarajona.

Babahoyo Emperador, Capítol ,Capítol \#2,Gran Daniel, Gran River, Perla verde

Montalvo Camino Real, Sabanetas

Vinces Azuay, Elisita, El Señor de los caballos

Baba Rock and Straw

Valencia Amores, Walnino

Mocache Complejo Emely

Pueblo Viejo Mango Tour

Fuente: MINTUR

Elaboración: Los Autores

La investigación de campo se guió con la siguiente ficha técnica:

Tabla 3

Ficha técnica de la Investigación

Población Objetivo Hoteles de Los Ríos


Tamaño de la muestra

Tipo de muestreo

Encuestas realizadas

Método de contacto

Tiempo de contacto

Fecha de realización del trabajo de campo
41

Censo

41

Directo

5:00 Minutos

8 de Abril del 2017 hasta el 11 de

Abril del 2017

Elaboración: Los Autores

\section{Resultados y Discusión}

Tabla 4.

En cuanto a su ubicación, ¿de qué tipo es su hotel?

\begin{tabular}{lcccc}
\hline Variable & $\begin{array}{c}\text { Frecuencia } \\
\text { Absoluta }\end{array}$ & $\begin{array}{l}\text { Frecuencia } \\
\text { Relativa }\end{array}$ & $\begin{array}{c}\text { Porcentaje } \\
\text { Válido }\end{array}$ & $\begin{array}{c}\text { Porcentaje } \\
\text { Acumulado }\end{array}$ \\
\hline Ciudad & 33 & $81 . \%$ & $81.0 \%$ & $81.0 \%$ \\
Aeropuerto & 0 & $0 \%$ & $0,0 \%$ & $81.0 \%$ \\
De playa & 0 & $0 \%$ & $0,0 \%$ & $81.0 \%$ \\
De naturaleza & 3 & $7.0 \%$ & $7.1 \%$ & $88.0 \%$ \\
Aparta- hotel & 0 & $0 \%$ & $0,0 \%$ & $88.0 \%$ \\
Albergue turístico & 0 & $0 \%$ & $0,0 \%$ & $88.0 \%$ \\
Hotel balneario & 5 & $12 . \%$ & $11.9 \%$ & $100.0 \%$ \\
Motel & 0 & $0 \%$ & $0,0 \%$ & \\
\hline Total & $\mathbf{4 1}$ & $\mathbf{1 0 0 \%}$ & $\mathbf{1 0 0 \%}$ & \\
\hline
\end{tabular}

\begin{tabular}{lccc}
\hline & Chi-Square Tests & & \\
\hline & Value & df & Asymp. Sig. (2-sided) \\
Pearson Chi-Square & $31.847^{\mathrm{a}}$ & 14 & .004 \\
Likelihood Ratio & 26.381 & 14 & .023 \\
N of Valid Cases & 41 & & \\
\hline
\end{tabular}

Fuente: Investigación

Elaboración: Los Autores 


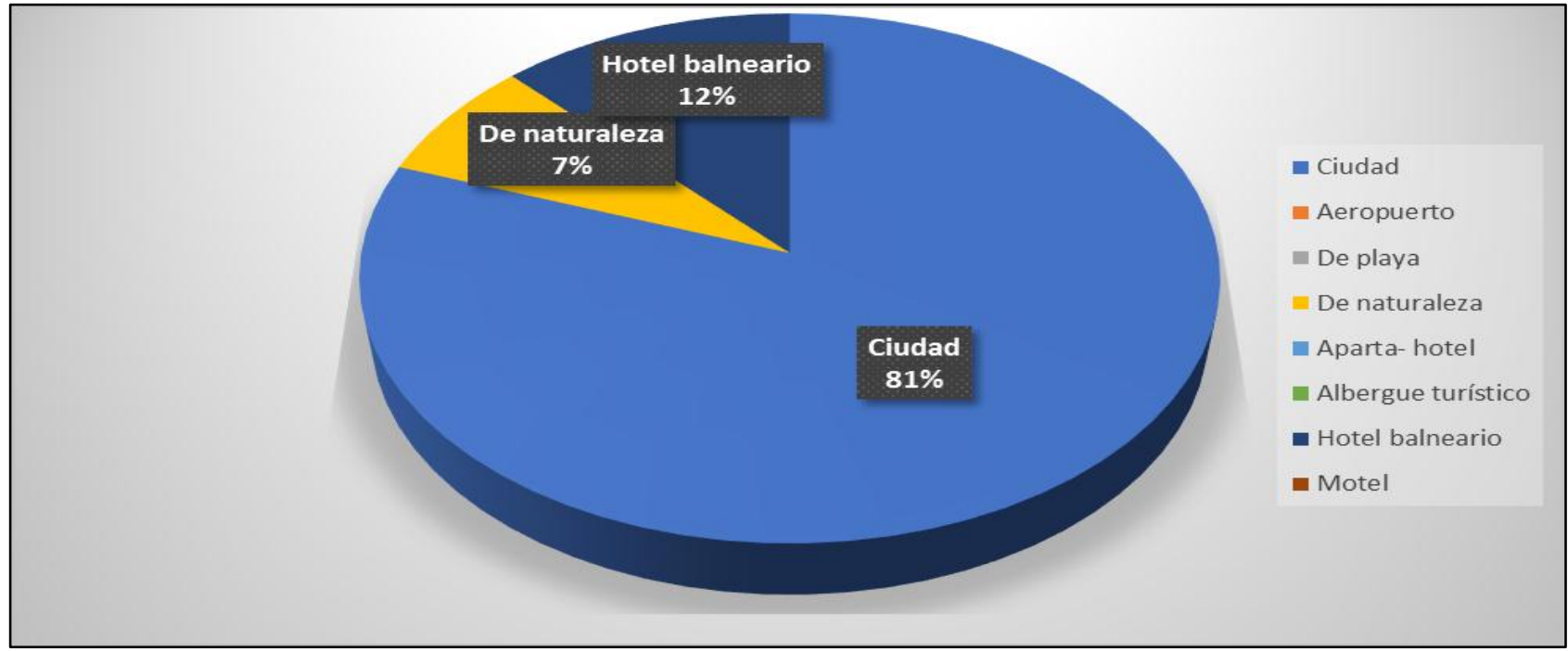

Figura 1. Ubicación de hoteles. Los Ríos, 2017

Discusión: De la información proporcionada por los censados, se observa que el $81 \%$ de los hoteles están ubicados en la ciudad. El 7 \% de los hoteles están rodeadas de vegetación; son de ubicación de naturaleza. Tal es el caso de los hoteles Palmar del Sol, Rancho San Vinicio y Riverside, del cantón Quevedo. Por otro lado, el 12\% son hoteles balnearios, que a más de su oferta hotelera, añaden balnearios naturales (ríos adecuados) y artificiales (piscinas) canchas deportivas, pistas de motos y caballos, entre otros, a sus productos hoteleros. Entre estos se cuentan: Camino Real (Montalvo), Rock and Straw (Baba), Complejo Emely (Mocache), Mango Tour (Pueblo Viejo) y El Señor de los caballos (Vinces). No se registraron hoteles de aeropuerto, de playa, Aparta- hoteles, Albergues turísticos o Moteles (estos últimos, mal llamados así en la provincia, en realidad son hostales, que no disponen de recepción, botones ni más de 50 habitaciones. Generalmente son ofrecidos por horas y no por noches, por lo que no entran en la caracterización de moteles). La hipótesis específica 1. "la ubicación o localización de los hoteles depende de la ciudad en la que se encuentra" se acepta porque, luego del análisis Chi-cuadrado, 
se evidencia que hay correlación entre las variables ciudad y Ubicación de los hoteles de Los Ríos, con un nivel de significancia del 5\%.

Tabla 5.

¿De qué tamaño es su hotel?

\begin{tabular}{lcccc}
\hline Variable & Frecuencia Absoluta & Frecuencia Relativa & $\begin{array}{c}\text { Porcentaje } \\
\text { Válido }\end{array}$ & $\begin{array}{c}\text { Porcentaje } \\
\text { Acumulado }\end{array}$ \\
\hline Pequeño & 41 & $100 \%$ & $100 \%$ & $100 \%$ \\
Mediano & 0 & $0 \%$ & $0 \%$ & $0 \%$ \\
Grande & 0 & $0 \%$ & $0 \%$ & $0 \%$ \\
Total & $\mathbf{4 1}$ & $\mathbf{1 0 0 \%}$ & $\mathbf{1 0 0} \%$ & $\mathbf{1 0 0} \%$ \\
\hline
\end{tabular}

Fuente: Investigación

Elaboración: Los Autores

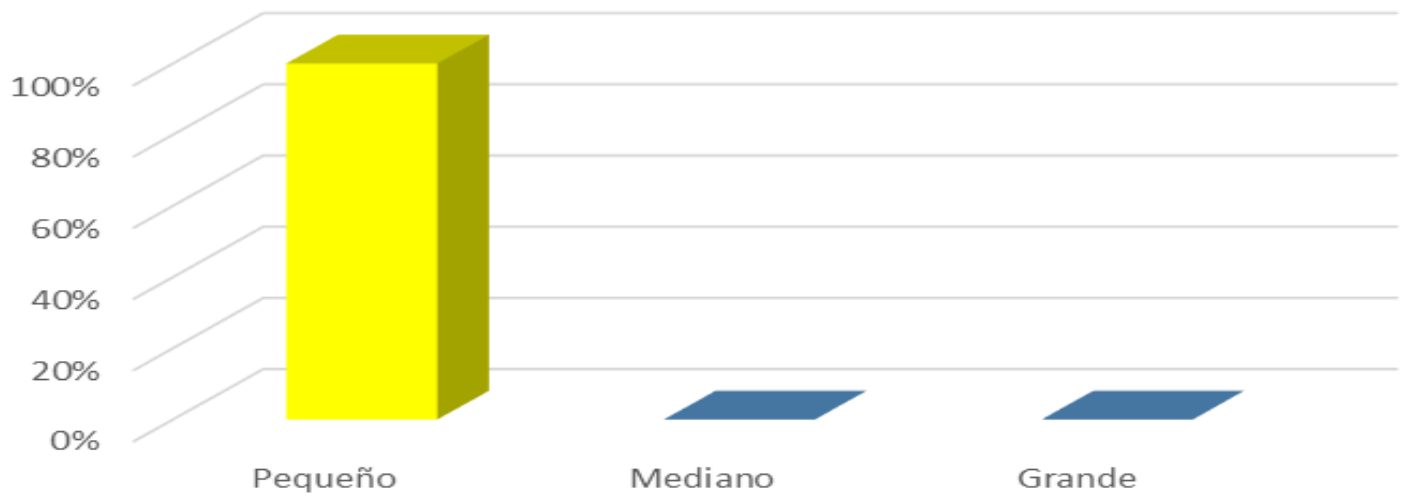

Figura 2. Tamaño de los hoteles. Los Ríos, 2016

Discusión: Los datos relativos a las respuestas de los censados evidencian que, en lo relativo a su tamaño, el $100 \%$ de los hoteles de la provincia de los Ríos pertenecen a la clase de tamaño pequeño: menos de 100 habitaciones. En ésta clasificación se omitió a los moteles, puesto que estos alojamientos rara vez se usan para estancias de más de tres horas.

Tabla 6.

En cuanto a su categoría, ¿cuántas estrellas asignó el MINTUR a su hotel?

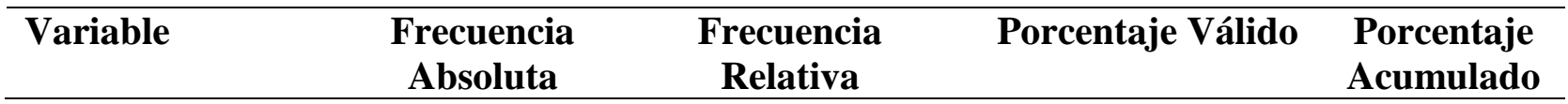




\begin{tabular}{|c|c|c|c|c|c|}
\hline Hoteles 2 estrellas & 11 & \multicolumn{2}{|c|}{$27 \%$} & $27 \%$ & $27 \%$ \\
\hline Hoteles 3 estrellas & 17 & \multicolumn{2}{|c|}{$44 \%$} & $44 \%$ & $71 \%$ \\
\hline Hoteles 4 estrellas & 12 & \multicolumn{2}{|c|}{$27 \%$} & $27 \%$ & $98 \%$ \\
\hline Hoteles 5 estrellas & 1 & \multicolumn{2}{|c|}{$2 \%$} & $2 \%$ & $100 \%$ \\
\hline Total & 41 & \multicolumn{2}{|c|}{$100 \%$} & $100 \%$ & $100 \%$ \\
\hline \multicolumn{6}{|c|}{ Chi-Square Tests } \\
\hline & & Value & df & \multicolumn{2}{|c|}{ Asymp. Sig. (2-sided) } \\
\hline Pearson Chi-Square & & $13.508^{\mathrm{a}}$ & 21 & \multicolumn{2}{|c|}{.890} \\
\hline Likelihood Ratio & & 16.568 & 21 & \multicolumn{2}{|c|}{.737} \\
\hline $\mathrm{N}$ of Valid Cases & & \multicolumn{4}{|l|}{41} \\
\hline
\end{tabular}

Fuente: Investigación

Elaboración: Los Autores

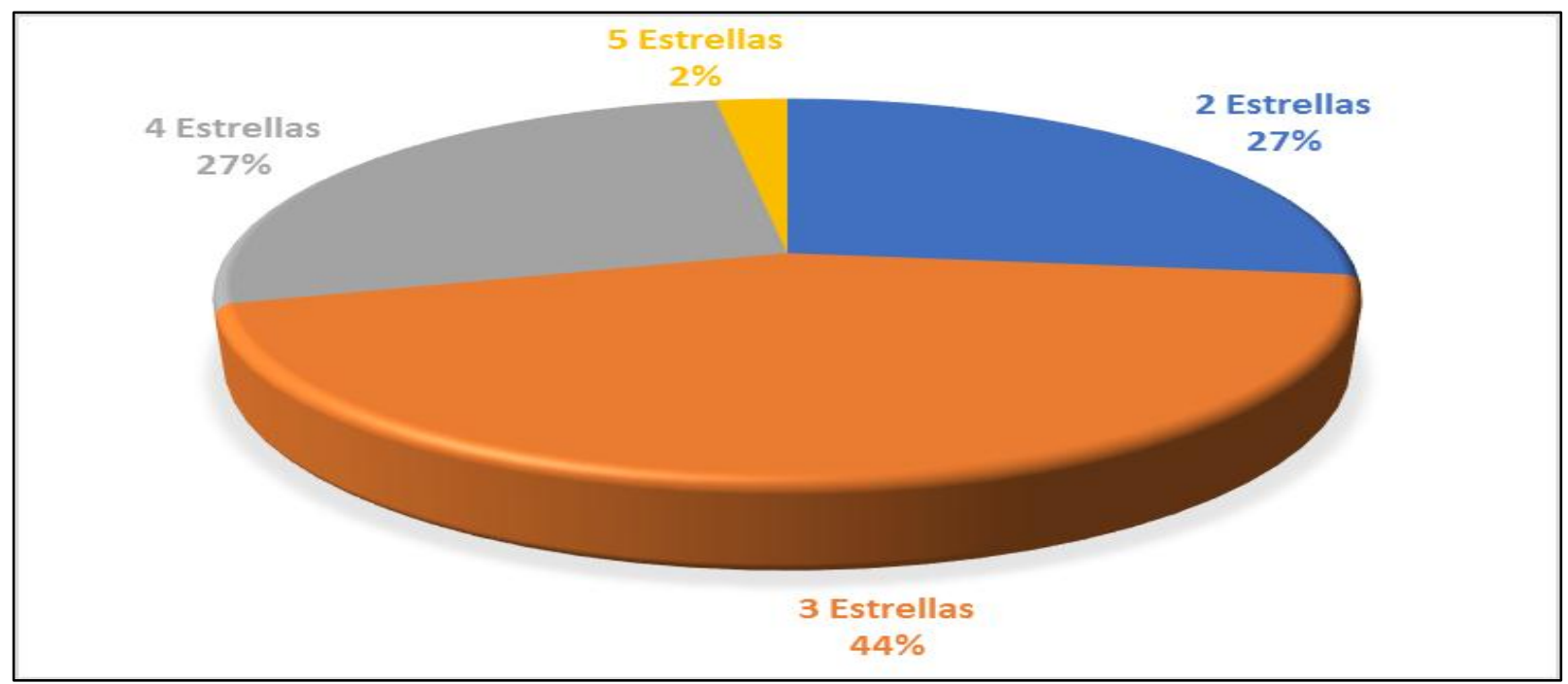

Figura 3. Categorización de los hoteles por estrellas. Los Ríos, Ecuador, 2017

Discusión: En cuanto a su categoría, medida en estrellas, -la clasificación más empleada en el sector hotelero mundial-, el mayor grupo de los hoteles de la provincia de los Ríos, (44\%), pertenece a la categorización de tres estrellas. Estos hoteles son muy utilizados por personas en viajes de negocios o turismo de negocios. Poseen habitaciones con baño y ducha, artículos de baños y tocador; cuentan con televisores, cable, internet y teléfonos. 
El $27 \%$ de los hoteles están categorizados en 4 estrellas. Se emplean para turismo de negocios y familiar. Las habitaciones están mejor equipadas con productos más refinados y elaborados. El servicio es más profesional, con servicios a la habitación las 24 horas.

Solo uno de los hoteles, El jade, está categorizado como de 5 estrellas, con el $2 \%$. Posee amplias instalaciones, chifa o restaurante chino de primera calidad (4 tenedores), ascensor, espacios amplios y lujosos. Su target es el estrato social alto. Las habitaciones cuentan con equipos de música, jacuzzis en los baños, televisión satelital, internet, servicio las 24 horas. También cuenta con un gimnasio.

La hipótesis específica 2. "La categoría de los hoteles de los Ríos está condicionada por la ciudad en la que laboran" acepta la hipótesis nula. El análisis chi-cuadrado evidencia que no hay correlación entre las variables ciudad y categoría de los hoteles de Los Ríos, con un nivel de significancia mayor al $5 \%$.

\section{Conclusiones}

El $81 \%$ de los hoteles de la provincia de los Ríos registrados en el MINTUR son de ubicación de ciudad. El $8 \%$ de los hoteles son de ubicación de naturaleza. Por otro lado, el 12\% de los hoteles son hoteles balnearios: a más de su oferta hotelera, añaden balnearios naturales y artificiales. No se registraron hoteles de aeropuerto, de playa, apart- hoteles, albergues turísticos o moteles, en el sentido técnico de su clasificación. La ubicación de los hoteles depende de la ciudad en la que se encuentran.

El $100 \%$ de los hoteles de la provincia de Los Ríos registrados en el MINTUR pertenece a la clase de tamaño pequeño.

El mayor grupo de los hoteles (44\%) de la provincia de los Ríos tiene la categorización de tres estrellas. Estos hoteles son muy utilizados por personas en viajes de negocios o turismo de 
negocios. El $27 \%$ de los hoteles están categorizados en 4 estrellas. Se emplean para turismo de negocios y familiar. Otro porcentaje igual (27\%) se categoriza como hoteles de 2 estrellas. Solo uno de los hoteles, que corresponde al 2\%, El Jade, está categorizado como de 5 estrellas. La categoría de los hoteles de los Ríos no está condicionada por la ciudad en la que laboran.

\section{Referencias bibliográficas}

Arcarons, R, Goitia, V. y González, N. (2012). La clasificación hotelera en la unión europea: un mercado poco común. $\quad$ Turismo. $\quad$ pp. $407, \quad 434$. www.papersdeturisme.gva.es/ojs/index.php/Papers/article/download.

Báez, A. (2005). Hotelería. México: Continental.

Bigné, J.; Moliner, M.; Vallet, T. y Sánchez, J. (1997): Un estudio comparativo de los instrumentos de medición de la calidad de los servicios públicos. Madrid: Revista española de investigación de marketing. Esic, septiembre, p. 33-52.

Cortina, F.; Martínez, A y Várela, B. Aproximación a la investigación del alojamiento privado de uso turístico. Estudios Turísticos, n. ${ }^{\circ} 151$ (2002) pp. 87-97.

Espino - Rodríguez, T., Gil - Padilla, A. (2014). Configuraciones en el sector hotelero basadas en las prioridades competitivas y su relación con el tamaño, categoría y el resultado organizativo. Universidad de Las Palmas de Gran Canaria, Vol. 15 N.o 1. Págs. 211 - 228. 2017. https://doi.org/10.25145/j.pasos.2017.15.013.

Esteban, A. y Reinares, E. Análisis del comportamiento de la demanda hotelera y su posible interferencia por el sistema de clasificación de la oferta. Revista Universidad Complutense de Madrid. http://www.ucm.es/BUCM/cee/doc/0024/03010024.htm Revisado [08/02/2008 17:20:31]. 
Gonzáles, R. (2003). Clasificación de hoteles. Buenos Aires: Trébol.

Lattin. (2007). La industria del hospedaje y el servicio de alimentos. Columbia: Planeta.

Mellinas, J., Martínez, S. M., Bernal, J. J. (2014). Uso de las redes sociales en los hoteles de la costa española. Investigaciones Turísticas ISSN: 2174-5609.

Organización Mundial del Turismo OMT (2017). Informe sobre la situación, perspectivas y oportunidades del turismo en el mundo. Madrid: World Tourism Organization.

Plan Estratégico de Desarrollo del Gobierno Provincial de Los Ríos. (2012). Babahoyo: GADPLR.

Proecuador (2014). Análisis sectorial del turismo. Quito: Dirección de Inteligencia Comercial e Inversiones.

Vallen, J. y Vallen., G. (2002). Check-in Check out. Dubuque, IA: Wm. C. Bros Publishers. 